\title{
Der Empowerment-Ansatz: unscharf, unbequem, unberechenbar - und unentbehrlich
}

\author{
The Enpowerment Concept: Fuzzy, Uncomfortable, Uncertain - and \\ Indipensable
}

\author{
Autor \\ J. Loss \\ Institut \\ Institut für Medizinmanagement und Gesundheitswissenschaften, Universität Bayreuth, Bayreuth
}

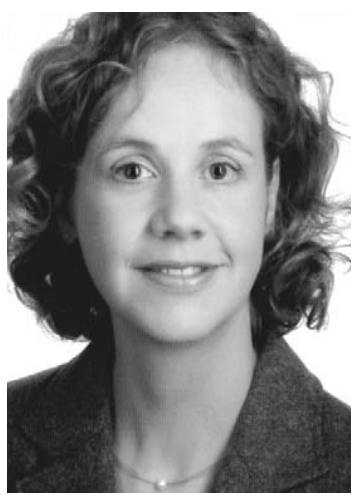

PD Dr. med. Julika Loss
Die Fülle an Publikationen, die man in der Gesundheitsförderungsliteratur zum Thema „Empowerment" im englischsprachigen Ausland findet, kontrastiert stark zu der eher zögerlichen Auseinandersetzung mit diesem Ansatz in Deutschland. Es muss allerdings eingeräumt werden, dass sich der Zugang zum Konzept des Empowerment nicht ohne Weiteres erschließt. So machen es die häufig sehr theoretischen Abhandlungen und Grundsatzartikel zu dem Thema den Gesundheitsförderern nicht unbedingt leicht, dieses Konzept in die Praxis umzusetzen. Zudem stammen viele Erfahrungen aus Entwicklungsländern: Hier ist der Ansatz des Empowerment angesichts häufig analphabetischer, entrechteter und unterdrückter Bevölkerungsgruppen einleuchtend. Aber müssen wir in unserer Wohlstandsgesellschaft mit demokratischer Grundordnung überhaupt Individuen „empowern“, um Gesundheitsförderung zu erreichen? Die Antwort ist eindeutig: Die Diskussion um die zunehmende Diskrepanz zwischen Arm und Reich auch in Deutschland, die wachsende Popularität des Themas der sozialen Ungleichheit von Gesundheit und Krankheit sowie der aktuelle Fokus auf Migranten und alte Menschen in der Prävention lassen das Empowerment auch hierzulande eindeutig und eindrücklich zu einer sinnvollen und wichtigen Strategie werden.

Bibliografie

DOI $10.1055 / \mathrm{s}-0028-1102958$ Gesundheitswesen 2008;

70: 713-714

(c) Georg Thieme Verlag KG Stuttgart · New York ISSN 0941-3790

\section{Korrespondenzadresse} PD Dr. med. J. Loss Institut für Medizinmanagement und Gesundheitswissenschaften Universität Bayreuth 95440 Bayreuth

julika.loss@uni-bayreuth.de
Allerdings zeigen die Erfahrungen aus dem internationalen Kontext auch: Nicht überall, wo „Empowerment" daraufsteht, steckt auch Empowerment darin. Das gilt auch für andere, ähnlich gelagerte Schlagwörter wie „Partizipation“. Bereits in den 60er Jahren sprach Sherry Arnstein im Kontext der Bürgerrechtsbewegung das Problem der „Alibi-Partizipation“ bzw. des „leeren Rituals der Partizipation“ an. Ähnliches findet sich auch weltweit im Umgang mit dem Ansatz des Empowerment. In der Praxis verbirgt sich oft hinter dem Schlagwort „Empowerment“ kaum mehr als die Vermittlung gesundheitsspezifischen Wis- sens, was dem wesentlich umfassenderen Ansatz nur wenig gerecht wird. Ein solcher „Etikettenschwindel“ geschieht allerdings selten bewusst und ist den Gesundheitsförderern meist nicht anzulasten. Vielmehr erweisen sich die Konzepte wie Empowerment und Partizipation bei ihrer Umsetzung als schwer fassbar, und Praktiker stoßen dabei oft an Grenzen. In ihrer Übersichtsarbeit zum Empowerment weisen Kliche und Kröger im vorliegenden Heft darauf hin, wie heterogen dieses Konzept sich in der internationalen Literatur darstellt. In Interviews, die ich mit australischen Gesundheitsförderern zum Thema Empowerment geführt habe, wurde unisono die Kritik an der Begrifflichkeit laut: der Terminus sei „fuzzy“, „cloudy“, „slippery“. Und das sagen Experten, in deren Muttersprache das Wort „Empowerment" entstanden ist. Wie viel schwerer haben es da deutschsprachige Gesundheitsförderer, die noch nicht einmal eine einheitliche Übersetzung haben finden können! Ermächtigung, Befähigung, Kompetenzsteigerung - diese und ähnliche Versuche der Übertragung greifen alle zu kurz, sodass es in Deutschland schwierig ist, sich selbst und anderen klarzumachen, was Empowerment eigentlich ist. Ganz zu schweigen davon, dass der im Englischen so geläufige und durchaus positiv besetzte Begriff „power“, um die es bei der Umsetzung von Bedürfnissen und Zielen der Gesundheitsförderung geht, im Deutschen („Macht“) vorrangig mit unangenehmen Konnotationen verbunden ist. „Verstärkung der Macht über ein Programm“, „Machterhalt“, „Machtverschiebung“ sind wohl keine Termini, mit denen man für das Konzept des Empowerment werben kann.

Ebenso gilt, dass in den letzten Jahren in der Gesundheitsförderung eine ganze Reihe von Begriffen aus dem Boden geschossen ist, deren Abgrenzung zum Empowerment-Konzept schwer ist, wie z.B. participation, capacity building, social capital oder assets. 
Um hier praxisnah grundlegende Fragen zu klären, hat sich im Januar dieses Jahres ein kleiner Kreis von deutschen Gesundheitsförderern in München getroffen, um insbesondere Strategien zur Umsetzung und Evaluation von Empowerment vorzustellen und zu diskutieren. Die Inhalte dieser Veranstaltung machen einen Großteil des vorliegenden Schwerpunktheftes aus. Dabei ging es auch darum, über den Tellerrand zu schauen: Impulse aus Australien (Loss \& Wise) wurden aufgenommen, vor allem aber der neuseeländische Empowerment-Experte Glenn Laverack mit seinen weltweit gesammelten Erfahrungen angehört. Erfreulicherweise zeigte sich, dass auch in Deutschland bereits einige gesundheitsförderliche Projekte laufen, die erfolgreich auf dem Empowerment-Ansatz aufbauen (Rütten et al., Trojan \& Nickel). Auch partizipative Ansätze der Qualitätsentwicklung können zum Empowerment der beteiligten Akteure eines gesundheitsförderlichen Programmes beitragen, wie Wright et al. beschreiben. Stärker umstritten war die Frage der angemessenen Evaluation dieses Konzepts: Welche Indikatoren sind zulässig, welche Verfahren und Methoden können eingesetzt werden (Trojan \& Nickel, Laverack, Loss \& Wise)? Hier zeigte sich, dass gelegentlich auch ungewöhnliche und innovative Wege beschritten werden müssen, um dem EmpowermentKonzept bei der Evaluation gerecht zu werden, z. B. in Form von qualitativen Verfahren.

Am Ende der Sitzung blieben viele Fragen offen - aber auch die Überzeugung, dass man auf diesem Gebiet in Deutschland noch viel mehr arbeiten muss. So stellt sich zum Beispiel die Frage, was Empowerment für spezifische Zielgruppen, z.B. Kinder und Jugendliche oder Ältere, bedeuten kann. Hierzu geben in dieser Ausgabe die Beiträge von Ravens-Sieberer et al. sowie Walter et al. Aufschluss. Eine weitere Originalarbeit beschäftigt sich mit der Messung von Empowerment bei Müttern (Sperlich).
Eines wird bei der Lektüre der vorliegenden Artikel deutlich: Empowerment ist schwer zu greifen. Empowerment ist unbequem, denn es wird dazu führen, dass die Experten etwas von ihrer „Macht“ abgeben und von ihren Prioritäten abrücken müssen oder sich gar mit Forderungen konfrontiert sehen, auf die sie nicht vorbereitet sind. Empowerment ist unberechenbar, denn der Ansatz erfordert auch ein „thinking outside the health box“. Empowerment ist langwierig, Empowerment ist aufwendig - aufwendiger als beispielsweise das Entwerfen, Gestalten und Drucken einer Hochglanzbroschüre. Deswegen kreisen viele Akteure in der Prävention und Gesundheitsförderung mit gewisser Ehrfurcht, aber auch mit Unbehagen, um das Empowermentkonzept. Und doch: Wir können nicht ohne Empowerment. Die Debatten um soziale Ungleichheit und wachsende Armut, das weitgehende Versagen rein aufklärerischer und edukativer Methoden sowie die ermutigenden internationalen wie auch zunehmend nationalen Erfahrungen lassen keinen anderen Schluss zu. Die Diskussion in eine größere Breite zu tragen und einen Erfahrungsaustausch anzustoßen, das soll daher auch mit diesem Schwerpunktheft erreicht werden - im Sinne einer „Ermutigung zum Empowerment“.

Ein besonderer Dank gilt dem Bayerischen Staatsministerium für Umwelt und Gesundheit, das mit einem Druckkostenzuschuss aus der Initiative Gesund.Leben.Bayern. einen freien Online-Zugang für diese Ausgabe auf der Homepage des ThiemeVerlags ermöglicht hat - und damit eine hoffentlich noch weitere Verbreitung des Themas Empowerment.

Dieses Heft kann in Gänze frei in elektronischer Form abgerufen werden unter http://www.thieme-connect.de/ejournals/toc/ gesu. 